\title{
Mutation status of epidermal growth factor receptor and clinical features of patients with combined small cell lung cancer who received surgical treatment
}

\author{
HONG-YANG LU ${ }^{1,4}$, WEI-MIN MAO ${ }^{1}$, QIAO-YUAN CHENG ${ }^{2}$, BO CHEN ${ }^{3}$, \\ JU-FEN CAI ${ }^{4}$, XIAO-JIA WANG ${ }^{1,4}$, ZENG WANG $^{5}$ and FA-JUN XIE ${ }^{4}$
}

\begin{abstract}
${ }^{1}$ Key Laboratory Diagnosis and Treatment Technology on Thoracic Oncology (esophagus, lung), Zhejiang Province, Zhejiang Cancer Hospital, Hangzhou, Zhejiang 310022; ${ }^{2}$ Department of Traditional Chinese Medicine, Zhejiang Institute for Food and Drug Control, Hangzhou, Zhejiang 310004; Departments of ${ }^{3}$ Pathology, ${ }^{4}$ Medical Oncology, and ${ }^{5}$ Pharmacology, Zhejiang Cancer Hospital, Hangzhou, Zhejiang 310022, P.R. China
\end{abstract}

Received January 9, 2012; Accepted March 27, 2012

DOI: $10.3892 / \mathrm{ol} .2012 .666$

\begin{abstract}
The mutation status of epidermal growth factor receptor (EGFR) is correlated with the response of tumors to EGFR tyrosine kinase inhibitors in non-small cell lung cancer (NSCLC), suggesting its usefulness as a biomarker in NSCLC. The incidence of EGFR mutation in NSCLC is higher in China than in the United States and European countries. There have been some case reports concerning cases of gefitinib-responsive small cell lung cancer (SCLC) with EGFR mutations. However, few large studies concerning the mutation status of SCLC patients have been performed. We detected EGFR mutations in exons 19 and 21 of 40 SCLC patients, three of whom had combined SCLC, from the Zhejiang Cancer Hospital using xTAG technology. Only two patients with combined SCLC had an EGFR mutation in exon 19. To determine the EGFR mutation status and clinical features of combined SCLC, we retrospectively analyzed the clinical features of seven patients with combined SCLC who had undergone surgical treatment in Zhejiang Cancer Hospital between 2007 and 2010. EGFR mutations in exons 19 and 21 were detected using the pyrosequencing assay. Of the seven patients with combined SCLCs, $71.4 \%$ were male, $71.4 \%$ were heavy smokers, most were over 60 years old and $71.4 \%$ of the cases were combined adeno-
\end{abstract}

Correspondence to: Dr Hong-Yang Lu, Key Laboratory Diagnosis and Treatment Technology on Thoracic Oncology (esophagus, lung), Zhejiang Province,Zhejiang Cancer Hospital, Hangzhou, Zhejiang 310022, P.R. China

E-mail: zjzlluhongyang@163.com

Abbreviations: EGFR, epidermal growth factor receptor; SCLC, small cell lung cancer; NSCLC, non-small cell lung cancer; RT-PCR, reverse transcription polymerase chain reaction; NCCN, National Comprehensive Cancer Network

Key words: epidermal growth factor receptor, gene mutation, pyrosequencing assay technology, combined small cell lung cancer carcinoma. Chemotherapy treatment and tumor stage were correlated with survival time. Of the seven cases, one had a mutation in exon 19 of EGFR in both the conventional SCLC and SCLC combined adenocarcinoma components. Combined SCLC commonly occurs in patients who are heavy smokers, male and over 60 years old, and most of the combined type cases are adenocarcinoma. The treatment of combined SCLC may be applied to cases of conventional SCLC. EGFR mutations may therefore occur in combined SCLCs, especially in SCLC combined adenocarcinoma in China.

\section{Introduction}

An epidermal growth factor receptor (EGFR) tyrosine kinase inhibitor (TKI) may be used as first-line therapy in patients with advanced non-small cell lung cancer (NSCLC) with EGFR mutations (1-5). The results of the INTEREST trial (6) suggest that gefitinib is able to provide similar overall survival to docetaxel in patients across a broad range of clinical subgroups and that EGFR biomarkers, including mutation status, may additionally identify which patients are likely to gain greatest progression-free survival (PFS) and overall response rate (ORR) benefit from treatment with gefitinib. Two hot spots of EGFR mutations are in-frame deletion at codons 747-749 (DEL) in exon 19 and a missense mutation at codon 858 (L858R) in exon 21. A case study concerning a Japanese patient with gefitinib-responsive small cell lung cancer (SCLC) reported that the patient had a deletion in exon 19 of EGFR (7). Another case study has reported that an American SCLC patient who had never smoked and who had an EGFR mutation responded to gefitinib (8). In China, there has also been a case report of a patient with SCLC who responded to gefitinib, but the status of the mutation is unknown (9). Therefore, the EGFR mutation status of SCLC is significant.

We detected EGFR exon 19 and exon 21 mutations in 40 SCLC patients, three of whom had combined SCLC, from the Zhejiang Cancer Hospital (Hangzhou, China) by xTAG technology. Only two of the combined SCLC patients showed an EGFR mutation in exon 19 (10). To identify the 
clinical features and incidence of EGFR mutations in cases of combined SCLC in China, we retrospectively investigated seven cases of combined SCLC which were treated surgically and detected mutations in EGFR exons 19 and 21 using a pyrosequencing assay. The combined components of these patients were not only adenocarcinoma but also squamous cell carcinoma. Specimens obtained during surgery more accurately reflect the pathological status of the tumor and all our specimens were from surgically resected tumors.

\section{Materials and methods}

Patient characteristics. Seven cases of combined SCLC from the Zhejiang Cancer Hospital (Hangzhou, China) between 2007 and 2010 were investigated. Of the patients with combined SCLC, $71.4 \%$ were male, $71.4 \%$ were heavy smokers, most were $>60$ years old and $71.4 \%$ of the cases were combined adenocarcinoma. None of the patients were clearly pathologically diagnosed prior to surgery. The specimens were obtained from surgically resected tumors. The histological diagnosis of combined SCLC was based on the standard criteria defined by the WHO classification. The patients were aged 47-74 years (median, 62), two were female and five were male. Two cases were stage IB, one was IIB and four were IIIA according to the seventh edition of the TNM classification for lung cancer. Two of the patients were non-smokers and five were heavy smokers. There were five cases of SCLC combined adenocarcinoma and two cases of SCLC combined squamous cell carcinoma (Fig. 1A). Most of the patients underwent lobectomy and lymph node dissection. Five patients received chemotherapy and two patients received no chemotherapy. Only one patient received thoracic radiotherapy (Table I). This study was approved by the ethics committee of the Zhejiang Cancer Hospital.

Pyrosequencing assay for gene mutation. We detected two examples of adenocarcinoma and SCLC combined adenocarcinoma components in case 6 . We also detected two examples of conventional SCLC and SCLC combined adenocarcinoma components in case 7 . In the other five patients, only one sample for each patient was detected. Genomic DNA was isolated and purified from formalin-fixed paraffin-embedded tissues using a GTpure FFPE Tissue DNA Extraction kit (GeneTech, Shanghai, China). For the amplification of fragments of exons 19 and 21 of the EGFR gene from isolated genomic DNA, we designed PCR amplification primers for pyrosequencing: EGFR-19, forward: 5'-GGATCCCAGAAGGTGAGAAAGTT-3'; EGFR-19, reverse biotinylated primer: 5'-GAGAAAAGGTGGGCCTG AGGT-3'; and EGFR-21, forward: 5'-GGGCATGAAC TACTTGGAGG-3'; EGFR-21, reverse biotinylated primer: 5'-TCCCTGGTGTCAGGAAAATG-3'. Each PCR assay contained forward and reverse primers (each 4 pmol), $2 \mu 1$ template DNA solution and 2 units hotstart Taq DNA Polymerase (Takara, Shiga, Japan) in a $40 \mathrm{ml}$ volume. The PCR conditions consisted of initial denaturation at $95^{\circ} \mathrm{C}$ for $3 \mathrm{~min} ; 50$ cycles of $95^{\circ} \mathrm{C}$ for $15 \mathrm{sec}$, annealing at $56^{\circ} \mathrm{C}$ for $30 \mathrm{sec}$ and $72^{\circ} \mathrm{C}$ for $30 \mathrm{sec}$; and final extension at $72^{\circ} \mathrm{C}$ for $5 \mathrm{~min}$. The PCR products were sequenced using the Pyrosequencing PyroMark ID system (Qiagen, Hilden, Germany) following the manufacturer's instructions, using the two pyrosequencing

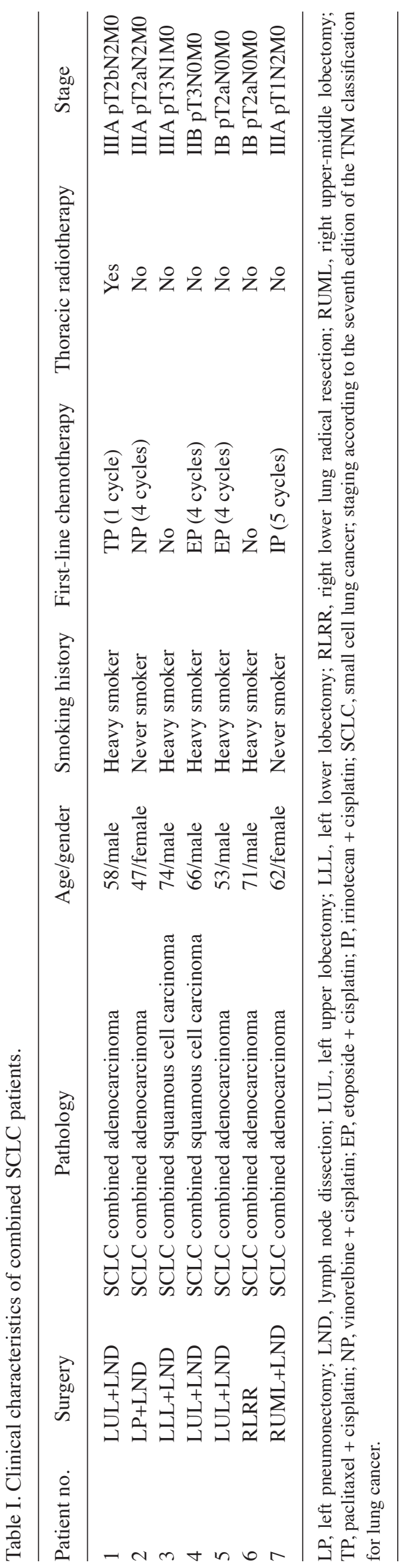


A

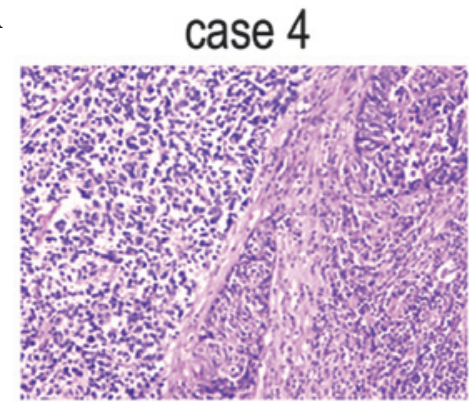

case $7-1$

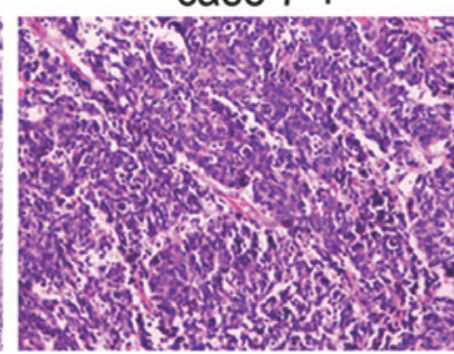

case $7-2$

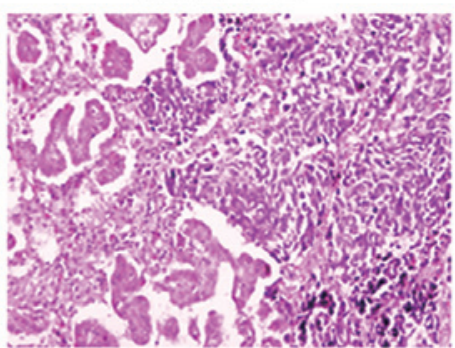

B
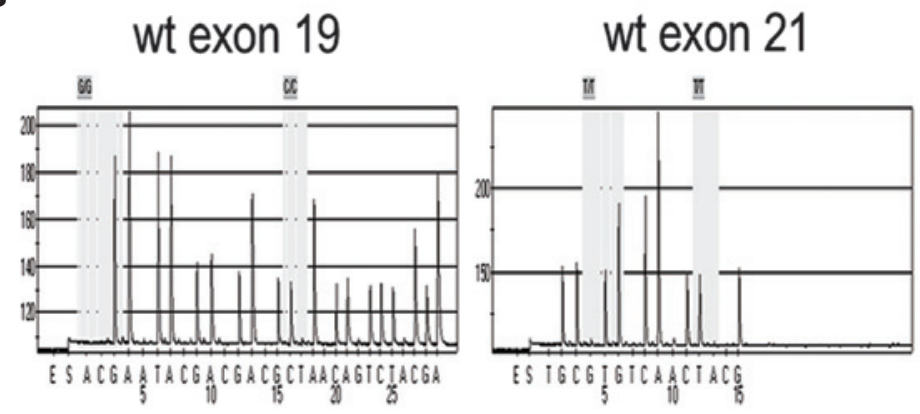

case 7 exon 19

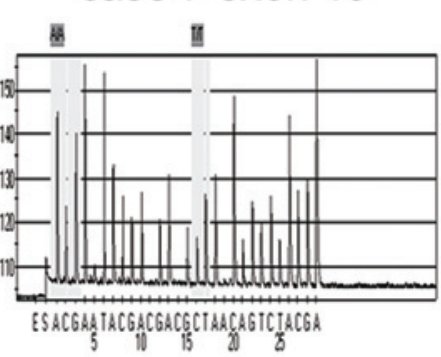

Figure 1. (A) Pathological results of case 4 were detected using HE staining, showing SCLC combined squamous cell lung cancer. Case 4 was a 66-year-old male with stage IIB pT3NOM0 squamous cell carcinoma. The pathological results of case 7-1 were detected by H\&E staining showing part of the conventional SCLC component. Case 7 was a 62-year-old female with stage IIIA pT1N2M0 SCLC combined adenocarcinoma. The pathological results of case 7-2 were detected using H\&E staining, showing the SCLC combined adenocarcinoma component. (B) Wt exon 19: Standard wild-type diagram of EGFR exon 19 (746-754). Sequencing target: G GAA TTA AGA GAA GCA ACA TCT CCG AAA. Wt exon 21: Standard wild-type diagram of L858R and L861Q in exon 21. Sequencing target: G CTG GCC AAA CTG. Case 7 exon 19: Testing diagram of EGFR exon 19 in case 7, mutation in exon 19 (codon 746-754) of EGFR both in the conventional SCLC and the SCLC combined adenocarcinoma components. H\&E, hematoxylin and eosin; SCLC, small cell lung cancer; EGFR, epidermal growth factor receptor; staging according to the seventh edition of the TNM classification for lung cancer.

Table II. Survival and EGFR mutation of combined SCLC patients.

\begin{tabular}{lccc}
\hline $\begin{array}{l}\text { Patient } \\
\text { no. }\end{array}$ & $\begin{array}{c}\text { Survival } \\
\text { (months) }\end{array}$ & $\begin{array}{c}\text { EGFR 19 } \\
\text { mutation }\end{array}$ & $\begin{array}{c}\text { EGFR 21 } \\
\text { mutation }\end{array}$ \\
\hline 1 & 26 & No & No \\
2 & $>31$ & No & No \\
3 & 10 & No & No \\
4 & $>18$ & No & No \\
5 & $>18$ & No & No \\
6 & $>47$ & No & No \\
7 & 22 & Yes & No \\
\hline
\end{tabular}

EGFR, epidermal growth factor receptor; SCLC, small cell lung cancer.

primers (5'-3' orientation): EGFR-19, TCCCGTCGCTATCAA; EGFR-21, AAGATCACAGATTTTGG. Pyrosequencing was performed using PyroMark Gold Q96 Reagents (Qiagen) containing enzyme and substrate mixture, dATP-S, dCTP, dGTP and dTTP.

Statistical analysis. The statistical significance of the mean values was determined using SPSS 13.0 (Chicago, IL, USA). $\mathrm{P}<0.05$ was considered to indicate a statistically significant result.

\section{Results}

The majority of the patients $(71.4 \%)$ received chemotherapy and most underwent more than 4 cycles. Cases 4 and 6 were alive at the end of their follow-up periods and cases 2 and 5 could not be followed up for longer than 31 and 18 months, respectively. Cases 3 and 6 did not receive chemotherapy and the survival time of case 6 (stage IB) was longer than that of case 3 (stage IIIA). The survival time of case 3 was shorter than that of the other stage IIIA patients who received chemotherapy (Table II). No mutations of exons 19 and 21 were observed, with the exception of case 7 . Case 7 was found to have a mutation in exon 19 (codon 746-754) of EGFR in both the conventional SCLC and SCLC combined adenocarcinoma components (Fig. 1B).

\section{Discussion}

Combined SCLC is defined as SCLC combined with an additional component that consists of any of the histological types of NSCLC, usually adenocarcinoma, squamous cell carcinoma or large cell carcinoma. SCLC accounts for approximately $15 \%$ of lung cancers (11). Combined SCLC has been reported to account for $1-3.2 \%$ of all SCLC cases $(12,13)$. However, $28 \%$ of SCLC patients who undergo surgical resection exhibit combined SCLC, with SCLC with large cell carcinoma being the most common, followed by adenocarcinoma and squamous cell carcinoma (14). Specimens obtained during surgery more accurately reflect the pathological features of 
the tumor than biopsy and malignant pleural effusion. In the study by Tatematsu et al, most of the specimens were obtained from biopsy (15). The specimens reported by Shiao et al include 10 computed tomography-guided biopsy specimens, 17 echo-guided aspiration specimens, 37 echo-guided biopsy specimens, one surgical lobectomy specimen and 11 malignant pleural effusion specimens (16), whereas, in the present study, all the specimens were obtained during surgery.

The incidence of the EGFR mutation in NSCLC is higher in China than in the United States and European countries $(17,18)$. Few large studies concerning the mutations status of patients with SCLC have been performed. EGFR mutations have been detected in five (4\%) of 122 Japanese patients with SCLCs, of which 15 cases were combined SCLCs. The patients with EGFR mutations were mainly in the light smoker and histological combined subtype. In three cases of the combined SCLC subtype, both components of adenocarcinoma and SCLC harbored an EGFR mutation (15). Fukui et al retrospectively investigated six resected cases of combined SCLC with an adenocarcinoma component in Japan to elucidate the clinicopathological parameters and detect the EGFR mutation status (19). With regard to EGFR, no specific mutation was detected in five of the six patients, whereas only one female patient who had never smoked had the same mutation in exon 21 (L858R) in both the SCLC and adenocarcinoma components (19). A prospective study on 76 specimens from patients with SCLC conducted between 2004 and 2009 at the National Taiwan University Hospital reported that two cases (2.6\%) tested positive for the EGFR mutation with reverse transcription polymerase chain reaction (RT-PCR) and direct sequencing and both cases were deletions in exon 19. Additionally, three patients were diagnosed with combined SCLC but showed no EGFR mutation in exon 19 or 21 (19). In our previous study, we detected EGFR mutations in exons 19 and 21 of 40 cases and two combined SCLCs with EGFR mutation in exon 19 (10). However, more combined SCLC cases are required to identify clinical features and detect EGFR exon 19 and 21 mutations in China. EGFR mutations were predictive of the response of single-agent TKIs, and EGFR gene copy number was also associated with response to TKIs, albeit with lower sensitivity and specificity (20). Therefore, in this study, we detected EGFR mutations instead of EGFR copy number. Compared with other genotyping and genetic detection methods, pyrosequencing technology is unique. Unlike hybridization-based assays, which may yield false-negative results, pyrosequencing produces a correct sequence even in the presence of a novel mutation. This is significant for microbiological applications. Another benefit of pyrosequencing is that the data is quantitative, thus it is possible to measure the relative amounts of alleles.

The seventh edition of the TNM classification was also cited in the National Comprehensive Cancer Network (NCCN) guidelines for SCLC (2011 version 1). Surgery may be used to treat NSCLC patients with stages IA, IB, IIA, IIB and IIIA disease, but only T1-2N0M0 SCLC patients may be considered for surgical treatment. Cases of T1-2N0M0 SCLC have been reported to account for less than 5\% of all SCLCs (21), thus few SCLCs can be treated surgically. It is difficult to diagnose combined SCLC by biopsy and the rarity of patients with combined SCLC makes it difficult to determine the optimal management and biological characteristics of this tumor. The treatment of combined SCLC was managed according to NCCN guidelines (Version 1.2011). There have been few studies concerning combined SCLC and more studies should be conducted to identify the clinical features of these patients. This study demonstrates that combined SCLC frequently occurs in patients who are heavy smokers, male and aged over 60 years and that most cases are combined adenocarcinoma. Case 3 did not receive chemotherapy and the survival time of this patient was shorter than that of the other stage IIIA patients who received chemotherapy. We suggest that chemotherapy is significant for combined SCLC and most of the patients in the present study received chemotherapy. Cases 3 and 6 did not receive chemotherapy, but the survival time of case 6 was longer than that of case 3 . The cause of the difference in survival time may be the different stages of case 3 (IIIA) and case 6 (IB). Thus, stage may affect survival time. Concurrent chemotherapy combined with mediastinal radiotherapy should be used to treat SCLC patients with lymph node metastasis following surgery and adjuvant chemotherapy and radiotherapy may be considered for stage IIIA NSCLC patients. Ideally, if the combined SCLC with lymph node metastasis is present after surgery, radiotherapy should be used in conjunction with chemotherapy. However, in our study, only one patient received radiotherapy.

In conclusion, EGFR mutations may occur in combined SCLCs, particularly in SCLC combined with adenocarcinoma in China.

\section{Acknowledgements}

This study was supported by the funds: No. Y2110004, Zhejiang Provincial Natural Science Foundation of China; No. 2010KYA035, Zhejiang Province Medical Science Fund Project of China; No. 2010ZA006, Zhejiang Province Traditional Medical Science Fund Project of China.

\section{References}

1. Mok TS, Wu YL, Thongprasert S, et al: Gefitinib or carboplatinpaclitaxel in pulmonary adenocarcinoma. N Engl J Med 361: 947-957, 2009.

2. Maemondo M, Inoue A, Kobayashi K, et al: Gefitinib or chemotherapy for non-small-cell lung cancer with mutated EGFR. N Engl J Med 362: 2380-2388, 2010.

3. Mitsudomi T, Morita S, Yatabe Y, et al: Gefitinib versus cisplatin plus docetaxel in patients with non-small-cell lung cancer harbouring mutations of the epidermal growth factor receptor (WJTOG3405): an open label, randomised phase 3 trial. Lancet Oncol 11: 121-128, 2010.

4. Rosell R, Gervais R, Vergnenegre A, et al: Erlotinib versus chemotherapy (CT) in advanced non-small cell lung cancer (NSCLC) patients (p) with epidermal growth factor receptor (EGFR) mutations: Interim results of the European Erlotinib Versus Chemotherapy (EURTAC) phase III randomized trial. J Clin Oncol 29 (Suppl): abs. 7503, 2011.

5. Zhou C, Wu YL, Chen G, et al: Erlotinib versus chemotherapy as first-line treatment for patients with advanced EGFR mutationpositive non-small-cell lung cancer (OPTIMAL, CTONG-0802): a multicentre, open-label, randomised, phase 3 study. Lancet Oncol 12: 735-742, 2011.

6. Douillard JY, Shepherd FA, Hirsh V, et al: Molecular predictors of outcome with gefitinib and docetaxel in previously treated non-small-cell lung cancer: data from the randomized phase III INTEREST trial. J Clin Oncol 28: 744-752, 2010.

7. Okamoto I, Araki J, Suto R, et al: EGFR mutation in gefitinib responsive small-cell lung cancer. Ann Oncol 17: 1028-1029, 2006. 
8. Zakowski MF, Ladanyi M, Kris MG, et al: EGFR mutations in small-cell lung cancers in patients who have never smoked. N Engl J Med 355: 213-215, 2006.

9. Qian J, Qin S, Tang Q, et al: Gefitinib in patients with advanced refractory small cell lung cancer contemporaneous with superior venacava syndrome. Chin Clin Oncol 10: 243-244, 2005.

10. Lu HY, Sun WY, Chen B, et al: Epidermal growth factor receptor mutations in small cell lung cancer patients who received surgical resection in China. Neoplasma 59: 100-104, 2012.

11. Govindan R, Page N, Morgensztern D, et al: Changing epidemiology of small-cell lung cancer in the United States over the last 30 years: analysis of the surveillance, epidemiologic, and end results database. J Clin Oncol 24: 4539-4544, 2006.

12. Mangum MD, Greco FA, Hainsworth JD, et al: Combined smallcell and non-small-cell lung cancer. J Clin Oncol 7: 607-612, 1989.

13. Fraire AE, Johnson EH, Yesner R, et al: Prognostic significance of histopathologic subtype and stage in small cell lung cancer. Hum Pathol 23: 520-528, 1992

14. Nicholson SA, Beasley MB, Brambilla E, et al: Small cell lung carcinoma (SCLC): a clinicopathologic study of 100 cases with surgical specimens. Am J Surg Pathol 26: 1184-1197, 2002.

15. Tatematsu A, Shimizu J, Murakami Y, et al: Epidermal growth factor receptor mutations in small cell lung cancer. Clin Cancer Res 14: 6093-6096, 2008
16. Shiao TH, Chang YL, Yu CJ, et al: Epidermal growth factor receptor mutations in small cell lung cancer: a brief report. J Thorac Oncol 5: 195-198, 2011.

17. Shigematsu H, Lin L, Takahashi T, et al: Clinical and biological features associated with epidermal growth factor receptor gene mutations in lung cancers. J Natl Cancer Inst 97: 339-346, 2005.

18. Wu YL, Zhong WZ, Li LY, et al: Epidermal growth factor receptor mutations and their correlation with gefitinib therapy in patients with non-small cell lung cancer: a meta-analysis based on updated individual patient data from six medical centers in mainland China. J Thorac Oncol 2: 430-439, 2007.

19. Fukui T, Tsuta K, Furuta K, et al: Epidermal growth factor receptor mutation status and clinicopathological features of combined small cell carcinoma with adenocarcinoma of the lung. Cancer Sci 98: 1714-1719, 2007.

20. Dahabreh IJ, Linardou H, Siannis F, et al: Somatic EGFR mutation and gene copy gain as predictive biomarkers for response to tyrosine kinase inhibitors in non-small cell lung cancer. Clin Cancer Res 16: 291-303, 2010.

21. Rostad H, Naalsund A, Jacobsen R, et al: Small cell lung cancer in Norway. Should more patients have offered surgical therapy? Eur J Cardiothorac Surg 26: 782-786, 2004. 